\title{
Enantioselective Synthesis of Versatile Stereogenic-at-P(V) Building Blocks via Hydrogen- Bond-Donor Catalysis
}

\author{
Katherine C. Forbes and Eric N. Jacobsen* \\ Department of Chemistry and Chemical Biology, Harvard University \\ Cambridge, MA 02138, USA.
}

*Corresponding author. Email: jacobsen@chemistry.harvard.edu

\begin{abstract}
The stereoselective synthesis of molecules bearing stereogenic phosphorus(V) centers represents an enduring challenge in organic chemistry. While stereospecific nucleophilic substitution at $\mathrm{P}(\mathrm{V})$ provides a general strategy for elaborating optically active $\mathrm{P}(\mathrm{V})$ compounds, existing methods for accessing the requisite chiral building blocks rely almost entirely on diastereocontrol using chiral auxiliaries. Catalytic, enantioselective methods for the synthesis of synthetically versatile stereogenic $\mathrm{P}(\mathrm{V})$ building blocks offer an alternative approach to stereogenic-at-P(V) targets without requiring stoichiometric quantities of chiral controlling elements. Herein, we report an enantioselective hydrogen-bond-donor-catalyzed synthesis of chlorophosphonamidates, and the development of these products as versatile chiral $\mathrm{P}(\mathrm{V})$ building blocks. We demonstrate that chlorophosphonamidates possess two leaving groups that can be displaced sequentially and stereospecifically to access a wide variety of stereogenic-at-P(V) compounds featuring diverse substitution patterns.
\end{abstract}

\section{Introduction}

Phosphorus(V) stereocenters are present in a wide assortment of important synthetic materials, including several recently developed pharmaceuticals (Figure 1A). The absolute stereochemistry at phosphorus is often directly associated with the biological activity of those molecules (1-7). Stereogenic-at-phosphorus compounds also serve as broadly useful ligands and catalysts in asymmetric organic synthesis $(8,9)$. While a variety of natural products bearing $P$-stereogenic centers have been identified (10), these molecules are not practical synthetic building blocks due to their sparsity. Thus, while the synthesis of compounds bearing $C$-stereogenic centers has historically drawn heavily on nature's chiral pool (11), access to $P$-stereogenic molecules relies entirely on de novo synthesis. Nucleophilic substitution at stereogenic $\mathrm{P}(\mathrm{V})$ centers can occur stereospecifically, thereby providing a powerful strategy for the synthesis of complex, optically active compounds from simple $\mathrm{P}(\mathrm{V})$ building blocks bearing one or more leaving groups attached to phosphorus $(9,11-13)$.

Effective methods for accessing stereogenic-at-phosphorus targets have relied primarily on the use of covalently attached chiral auxiliaries to achieve diastereocontrol, and a variety of chelating auxiliaries have been developed successfully for this purpose (Fig. 1B) (14-22). Their applicability depends on the ability to achieve stereospecific displacement of the auxiliary to forge $\mathrm{P}(\mathrm{V})$ stereocenters with absolute stereocontrol. Among noteworthy recent advances using the chiral auxiliary approach, Baran and co-workers reported the development of highly reactive oxathiaphospholane-sulfide building blocks $(21,22)$. The propensity of the $\mathrm{P}-\mathrm{S}$ bonds in these building blocks to undergo substitution by both alcohols and organometallic reagents was 

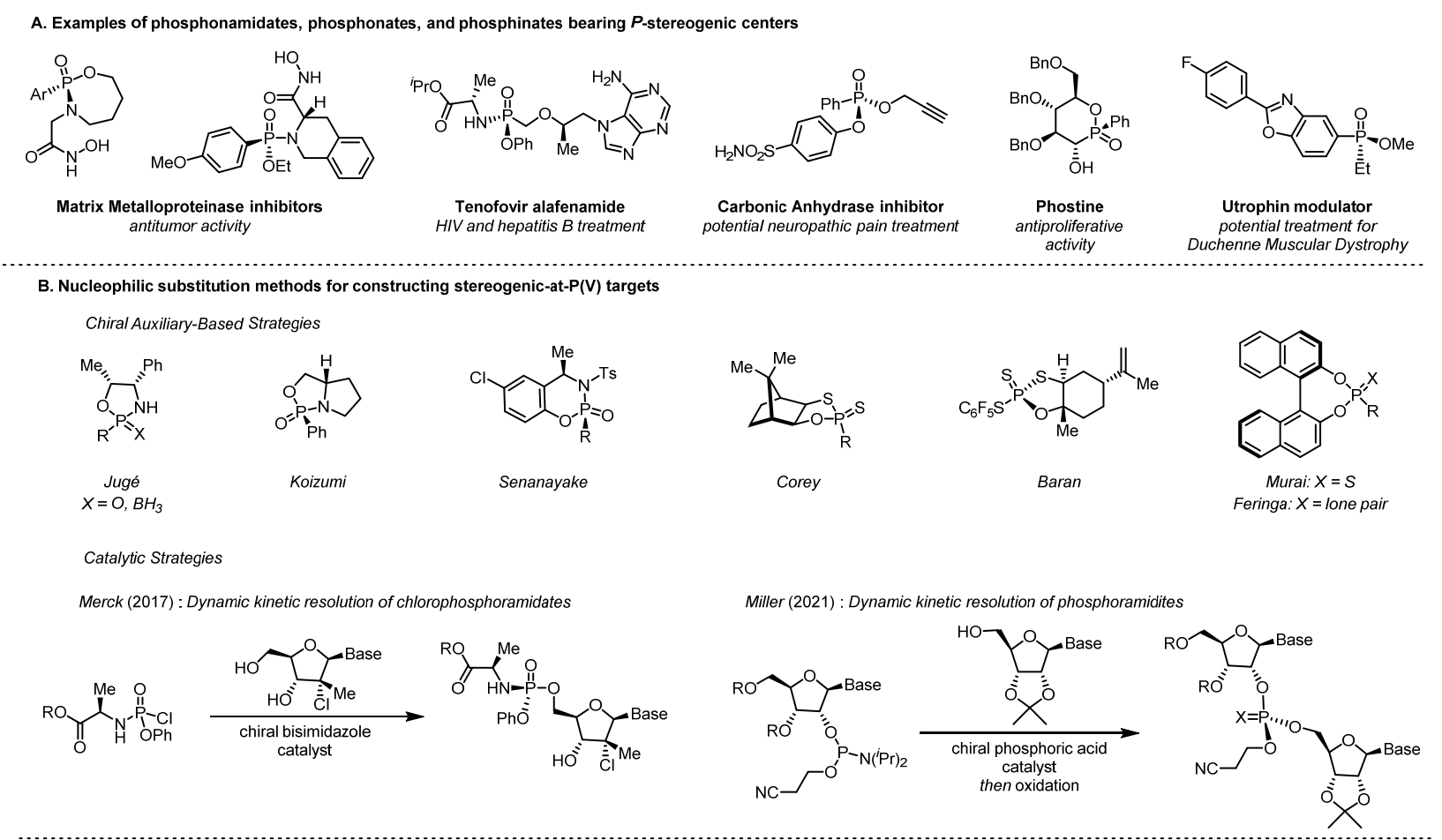

C. This work: catalytic synthesis of chiral $P(V)$ building blocks via hydrogen-bond-donor catalysis

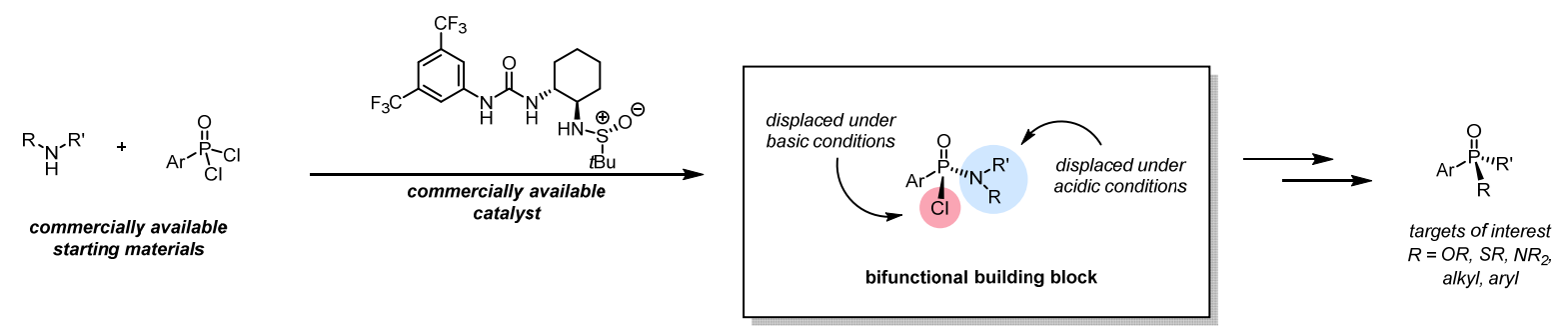

Fig. 1. Methods for accessing stereogenic $\mathbf{P}(\mathrm{V})$ targets. (A) Representative bioactive compounds bearing $P$ stereogenic centers. (B) Synthetic approaches. (C) A general approach to chiral $\mathrm{P}(\mathrm{V})$ building blocks via enantioselective catalysis/stereospecific substitution.

demonstrated and enables the synthesis of a variety of stereogenic-at-P(V) compounds, ranging from oligonucleotides to chiral phosphine oxides.

Despite the significant advances in the stereoselective synthesis of chiral $\mathrm{P}(\mathrm{V})$ compounds by the chiral auxiliary approach, there is both practical and fundamental motivation for developing asymmetric catalytic strategies toward these targets. In that vein, there have been important recent breakthroughs (Fig. 1B). Merck developed a chiral bisimidazole catalyzed synthesis of phosphoramidate prodrugs through the diastereoselective addition of nucleosides to chlorophosphoramidates, proceeding via a cooperative mechanism of covalent activation of $\mathrm{P}(\mathrm{V})$ and general-base activation of the alcohol nucleophile (23). An alternative approach was demonstrated by Miller and co-workers in the catalytic, stereodivergent synthesis of $P$-stereogenic oligonucleotides from phosphoramidites via chiral phosphoric acid catalysis (24). Finally, in work that appeared as this study was being completed, Dixon and co-workers reported a catalytic, enantioselective desymmetrization of diaryl phosphonate esters by substitution with orthosubstituted phenols (25). While high levels of stereoselectivity were achieved in these catalytic, nucleophilic substitution reactions, each is limited to a narrow class of nucleophiles that are not 
further displaced. We conceived that the catalytic, enantioselective installation of a nucleophile that could further serve as a leaving group for stereospecific substitution at $\mathrm{P}(\mathrm{V})$ could provide a generalizable strategy for the synthesis of chiral $\mathrm{P}(\mathrm{V})$ targets with the broad synthetic scope of state-of-the-art auxiliary approaches while avoiding the need for the stoichiometric use of chiral control elements.

We selected chlorophosphonamidates as potential targets of an enantioselective catalytic approach (Fig. 1C). The chloride and amino groups on $\mathrm{P}(\mathrm{V})$ possess orthogonal reactivity that might permit sequential and stereospecific displacement en route to chiral $\mathrm{P}(\mathrm{V})$ targets bearing a broad range of substitution patterns. Given that $\mathrm{P}-\mathrm{Cl}$ bonds in particular are susceptible to substitution by a wide variety of nucleophiles (26-28), chlorophosphonamidates would be highly versatile precursors to a multitude of $\mathrm{P}(\mathrm{V})$ frameworks. We report here the development of an enantioselective method for the synthesis of chlorophosphonamidate intermediates using a commercially available hydrogen-bond-donor catalyst, and the application of these $\mathrm{P}(\mathrm{V})$ building blocks to the synthesis of $\mathrm{P}(\mathrm{V})$ compounds featuring diverse substitution patterns.

We recognized that a most concise enantioselective synthesis of chlorophosphonamidates would be realized via a catalytic desymmetrization reaction of phosphonyl dichlorides with amines. Dual-hydrogen-bond-donor catalysts have been applied broadly and successfully to promote stereoselective nucleophilic substitution reactions via chloride-abstraction pathways (2932 ), and we hypothesized that this reactivity principle could serve to activate one of the two enantiotopic chlorides of a phosphonyl dichloride electrophile toward displacement by an amine. Phenyl phosphonic dichloride 2a was selected as a model substrate in reactions with various amine nucleophiles and potential chiral catalysts (Fig. 2). For ease of isolation and analysis, the chlorophosphonamidate products $\mathbf{3}$ were quenched with sodium methoxide at low temperature to produce the corresponding phosphonamidate 4a. After systematic evaluation of a series of chiral dual H-bond-donor catalysts and amine nucleophiles, the sulfinamido urea 1a $(33,34)$ was found to promote the nucleophilic substitution by diisoamylamine in $95 \%$ enantiomeric excess (ee) and quantitative yield (Fig. 2A, see supplementary materials for optimization studies). Multiple equivalents of amine were required to attain full conversion of $\mathbf{2 a}$, as the amine functions both as a nucleophile and as a stoichiometric Brønsted base to trap the $\mathrm{HCl}$ byproduct produced in the reaction. Examination of the role of catalyst structure revealed the importance of both the H-bond donor and the sulfinamide group in promoting high enantioselectivity. Whereas sulfinamido urea 1a and its thiourea analog $\mathbf{1 b}$ proved similarly effective as catalysts, the sulfinamide $\mathbf{1 d}$ lacking the H-bond-donor motif induced little acceleration above the uncatalyzed rate $(83 \%$ vs. $64 \%$ yield after $24 \mathrm{~h}$ ) and afforded only racemic product. The sulfinamido urea 1c epimeric to $1 \mathrm{a}$ also induced severely diminished enantioselectivity. Arylpyrrolidino (thio)ureas such as $\mathbf{1 e - g}$, which have proven useful in a wide range of asymmetric anion-binding pathways (35) but that lack the sulfinamide moiety, were catalytically active but generally poorly effective with respect to enantiocontrol. The enantioselectivity of the substitution was also closely tied to the identity of the amine, with diisoamylamine undergoing reaction with distinctly superior results relative to any of the other nucleophiles examined (Fig. 2B). Beyond a beneficial effect of distal alkyl branching, it is difficult to discern any straightforward correlation between the steric or electronic properties of the amine and enantioselectivity in the substitution reaction. It is likely that the properties of the dialkylammonium chloride byproducts play a critical and complex role in influencing the observed 


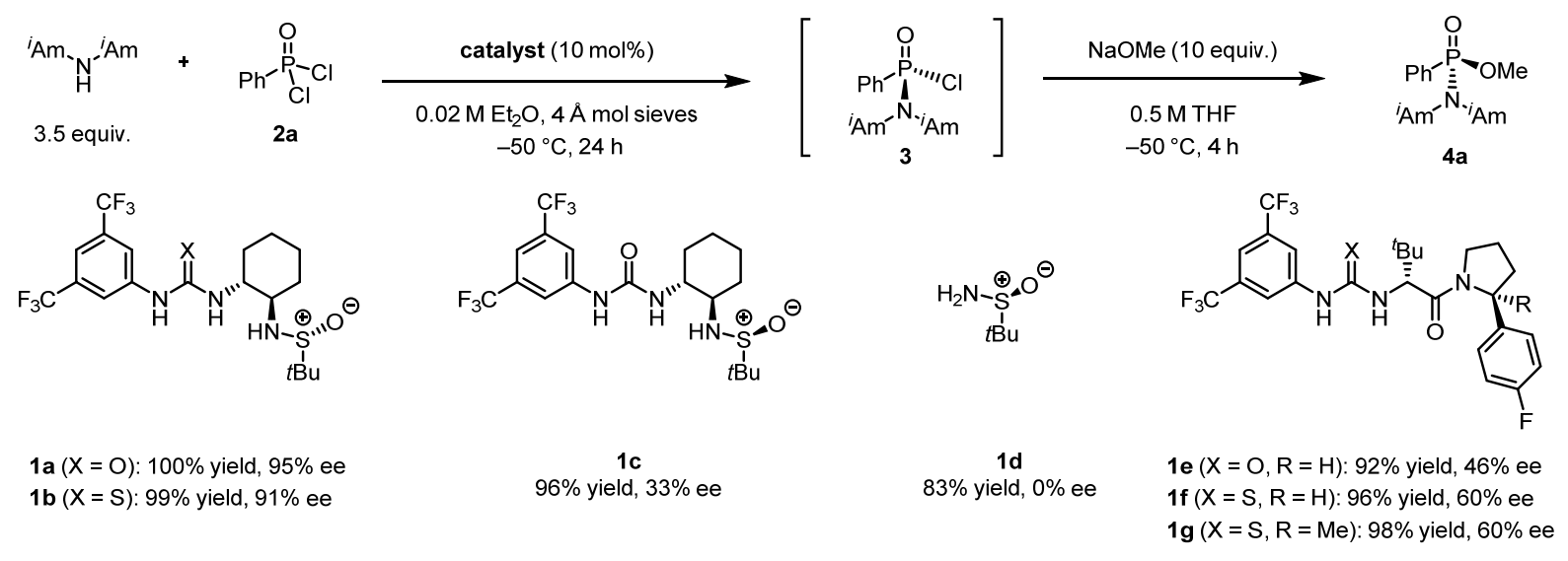

B. Effect of Amine Structure

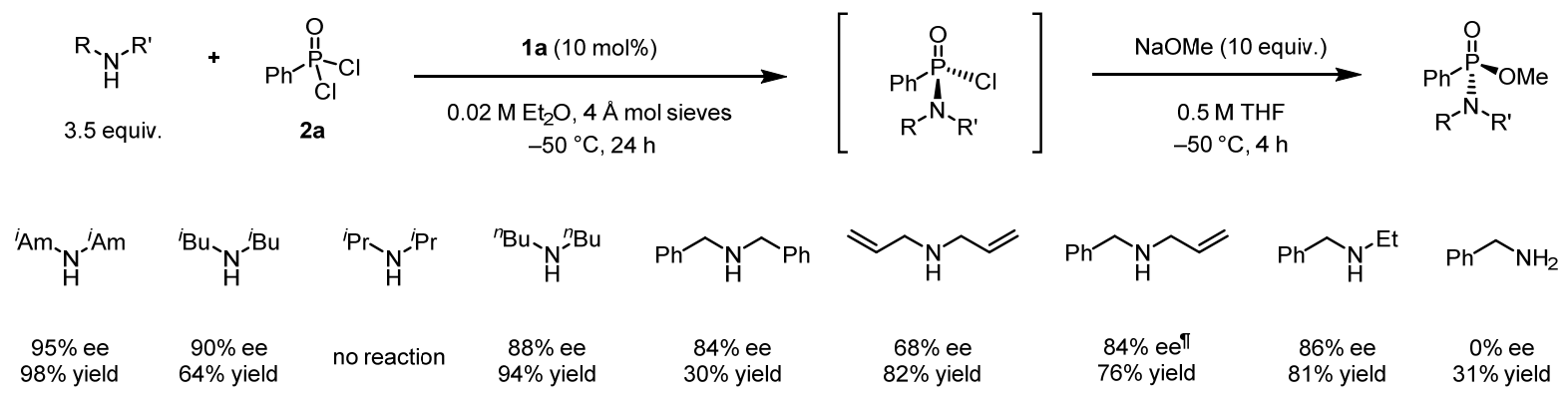

Figure 2. Optimization studies. Yield values reflect product quantification by ${ }^{31} \mathrm{P}$ NMR relative to an internal standard. (A) Catalyst optimization for enantioselective reaction of diisoamylamine with phenyl phosphonic dichloride. Reactions were carried out on a $0.06 \mathrm{mmol} \mathrm{scale}$. (B) Optimization of amine structure for enantioselective substitution reaction with phenyl phosphonic dichloride. Reactions were carried out on a 0.06 mmol scale. ${ }^{\text {I }}$ Reaction performed at $-40{ }^{\circ} \mathrm{C}$ for $48 \mathrm{~h}$.

enantioselectivity, as soluble tetraalkylammonium chloride salts are potent inhibitors of anionbinding H-bond-donor catalysts and were also shown to promote a racemic reaction between $\mathbf{2 a}$ and diisoamylamine (Tables S4-S5). Epimerization of chlorophosphonamidate 3 was not observed under the catalytic conditions, even in the presence of added tetrabutylammonium chloride.

High levels of enantioselectivity were achieved in the reaction of a variety of aryl phosphonyl dichlorides with diisoamylamine (Fig 3A). Notably, substrates bearing arenes with either electron-withdrawing or electron-donating substituents underwent substitution with consistently high levels of enantioselectivity $(\mathbf{4 b}-\mathbf{g})$. However, alkyl phosphonyl dichlorides yielded product with low levels of enantioselectivity under analogous conditions (see supplementary materials for examples). 


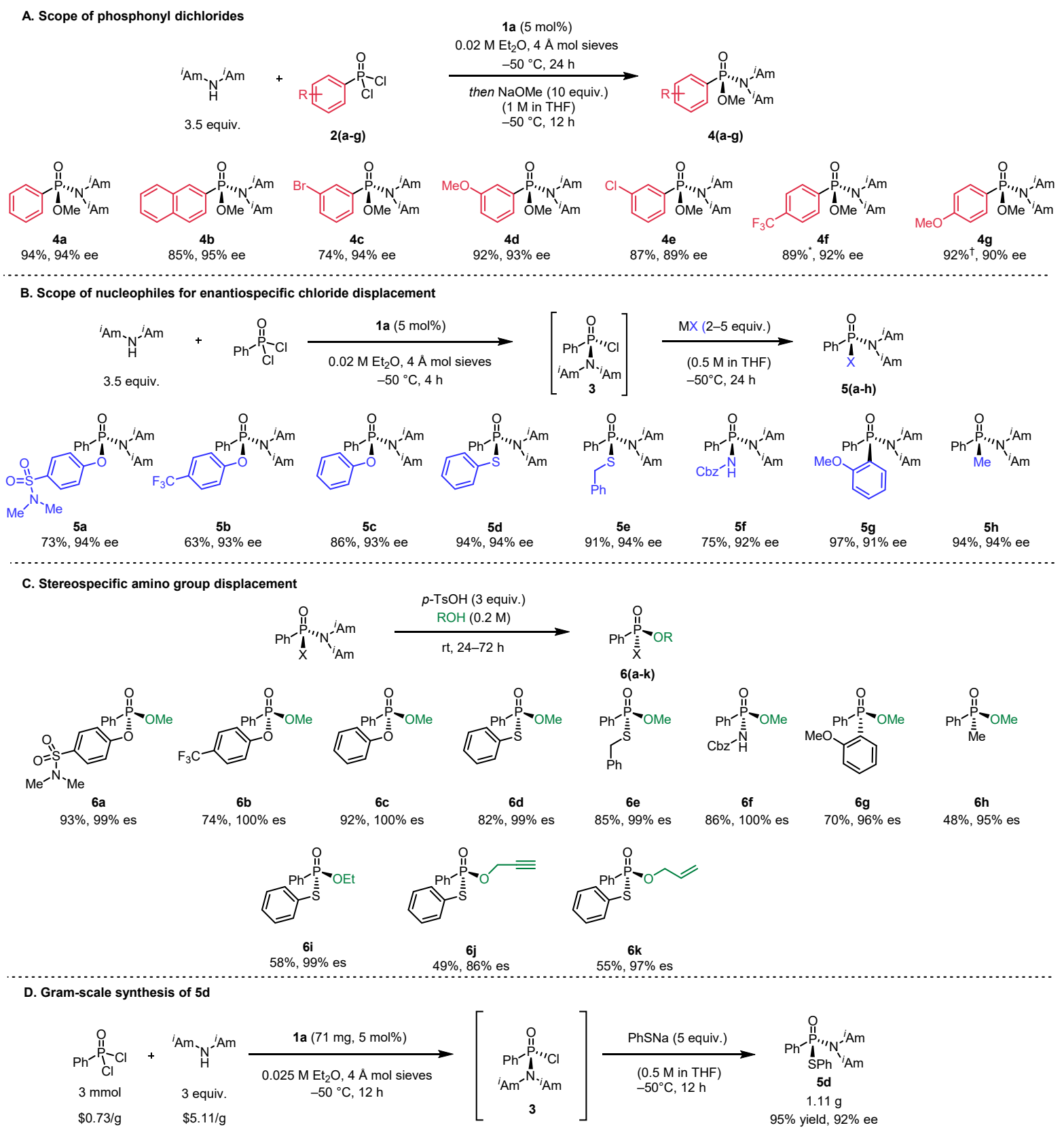

Fig. 3. Scope of enantioselective addition of diisoamylamine to aryl phosphonyl dichlorides and stereospecific elaborations. All yield values correspond to chromatographically purified, isolated products. (A) Substrate scope of addition of diisoamylamine to aryl phosphonyl dichlorides catalyzed by 1a. Reactions were carried out on $0.2 \mathrm{mmol}$ scale. The absolute stereochemistry of the products was assigned based on the X-ray crystal structure of $\mathbf{1 0}$ and the known optical rotation of 8a (Fig. 4, see supplementary materials). (B) Scope of nucleophiles for enantiospecific substitution with 3. (C) Enantiospecific displacement of the diisoamylamino group with alcohols. See supplementary materials for reaction conditions. (D) Gram-scale synthesis of 5d. Prices from Thermo Fisher Scientific (February, 2022). *Reaction was carried out at $-78{ }^{\circ} \mathrm{C}$ with $20 \mathrm{~mol} \%$ catalyst loading. $\dagger$ Reaction was carried out at $-40^{\circ} \mathrm{C}$ with 4.5 equivalents of diisoamylamine. 
The products of the enantioselective reactions feature two chemically distinct leaving groups on phosphorus that could be selectively and stereospecifically displaced to afford access to multiple classes of chiral $\mathrm{P}(\mathrm{V})$ compounds. We first explored the scope of nucleophiles capable of enantiospecific displacement of the remaining chloride (Fig. 3B). Reaction of $\mathbf{3}$ with alkoxides, phenoxides, thiolates, carbamides, and Grignard reagents afforded the desired products with high levels of enantiospecificity in all cases $(\mathbf{5 a}-\mathbf{h})$. The substitution reactions could be performed with or without isolation of $\mathbf{3}$ from the prior enantioselective catalytic step (see supplementary materials for details). Notably, we found that the reactions could be scaled up without loss of enantioselectivity or yield; thus, the synthesis of $\mathbf{5 d}$ was performed by the one-pot procedure on 3 mmol scale with $5 \mathrm{~mol} \%$ catalyst, affording 1.11 grams of product in $95 \%$ yield and $92 \%$ ee (Fig. $3 \mathrm{D})$.

The products of the chloride-displacement reactions could be further elaborated to afford alkoxy-substituted $\mathrm{P}(\mathrm{V})$ compounds via an acid-mediated stereoinvertive displacement of the diisoamylamino group (Fig. 3C). Substitution of $\mathbf{5 a}-\mathbf{h}$ with methanol yielded a variety of enantioenriched phosphonates, phosphinates, and phosphonamidates $(\mathbf{6 a}-\mathbf{h})$ with nearly complete enantiospecificity observed in every case. The slightly diminished stereospecificity observed with $\mathbf{5 g}$ and $\mathbf{5 h}$ is consistent with prior observations $(14,16)$. Substitution with other primary alcohols proceeded with varied but generally high levels of enantiospecificity $(\mathbf{6 i}-\mathbf{k})$.

The phosphonate ester and thioester products $\mathbf{6 b}$ and $\mathbf{6 d}$ possess further readily displaceable substituents that render them as useful synthetic building blocks for further elaboration to chiral $\mathrm{P}(\mathrm{V})$ compounds. For example, phosphonate thioester $\mathbf{6 d}$ underwent reaction with functionally complex alcohols to furnish the corresponding phosphonylated biomolecules with high levels of stereospecificity (7a-c, Fig. 4A). These substitutions are performed under Brønsted-acid-free conditions using little-or-no excess of the alcohol reagent, highlighting the utility of $\mathbf{6 d}$ for the phosphonylation of precious or acid-sensitive alcohols. Phosphonate $\mathbf{6 b}$ underwent efficient substitution with Grignard reagents with displacement of the electron-deficient aryloxide to yield highly enantioenriched phosphinate esters, known precursors to chiral phosphine oxides (Fig. 4B) (22). This three-step route to phosphinate esters was applied to the synthesis of $(+)$-SMT022332, a utrophin modulator developed as a potential treatment for Duchenne Muscular Dystrophy (36-38). An analogue of (+)-SMT022332 was previously accessed in $83 \%$ ee and 5\% overall yield using a chiral auxiliary-based approach (4). Subjection of phosphonyl dichloride 9 to the optimized conditions for the enantioselective substitution yielded phosphonamidate 10, which was characterized crystallographically (Fig. 4C). Subsequent methanolysis and phenol displacement furnished (+)-SMT022332 (12) in 94\% ee and 43\% overall yield over 3 steps. 


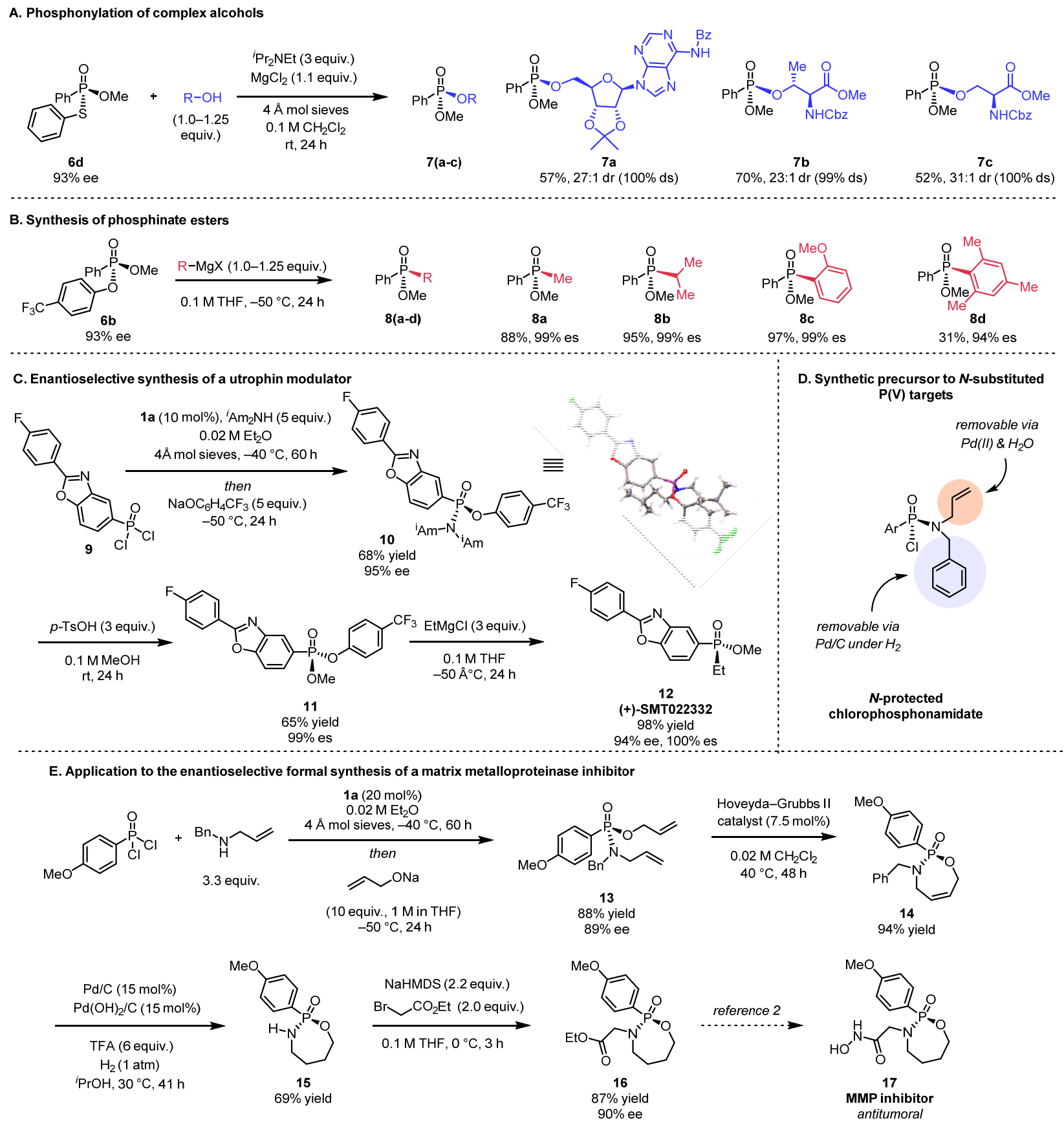

Fig. 4. Application to the synthesis of chiral $P(V)$ targets. All yield values refer to chromatographically purified, isolated products. (A) Stereospecific phosphonylation of precious alcohols with 6d. Reactions were carried out on $0.1 \mathrm{mmol}$ scale. (B) Stereospecific addition of Grignard reagents to $\mathbf{6 b}$ for the synthesis of enantioenriched phosphinate esters. Absolute stereochemistry of $\mathbf{8 a}$ was determined by comparison of optical rotation to literature value; others assigned by analogy. Reactions run on $0.05-0.1 \mathrm{mmol} \mathrm{scale}$. (C) Application of method to the enantioselective synthesis of (+)-SMT022332. Yield values refer to isolated yields. Absolute stereochemistry of $\mathbf{1 0}$ assigned by the depicted X-ray crystal structure, and of $\mathbf{1 2}$ by comparison of the optical rotation to the literature value. (D) Orthogonally $N$-protected chlorophosphonamidate. (E) Formal synthesis of a matrix metalloproteinase inhibitor. TFA $=$ trifluoroacetic acid. 
In addition to serving as versatile synthetic building blocks, phosphonamidates are often synthetic targets themselves $(2,3,5,6,39-43)$, and general access to these compounds by the catalytic procedure would be desirable. However, the structural requirements on the amine for achieving high enantioselectivity in catalytic reaction impose restrictions to the $\mathrm{N}$-substituents that can be introduced directly (Fig. 2B). We therefore sought to identify amine derivatives that participate successfully in the enantioselective reaction while bearing orthogonally cleavable $\mathrm{N}$ protecting groups that might provide centralized access to a variety of substituted phosphonamidates (Fig. 4D). High enantioselectivity was obtained using $N$-allyl benzylamine in the substitution reaction under modified conditions. The benzyl group and the allyl group on the chlorophosphonamidate products can each be cleaved successively, enabling their sequential replacement (see supplementary materials) (44-48). This strategy was exploited in the synthesis of phosphonamidate 17, a matrix metalloproteinase (MMP) inhibitor with demonstrated anticancer activity (Fig. 4E) (2). Phosphonic dichloride $2 \mathrm{~h}$ effectively underwent the catalytic reaction with $\mathrm{N}$-allylbenzylamine to produce, after quenching with allyl alkoxide, phosphonamidate $\mathbf{1 3}$ in $89 \%$ ee and $88 \%$ yield. Phosphonamidate 13 was elaborated over three steps to afford cyclic phosphonamidate 16 in $90 \%$ ee, completing the enantioselective formal synthesis of MMP inhibitor 17. We anticipate that $\mathrm{N}$-allyl benzylamine's versatility as an " $-\mathrm{NH}_{2}$ " equivalent may enable access to a wide variety of phosphonamidate targets.

In this study, enantioenriched chlorophosphonamidates are introduced as a new class of chiral $\mathrm{P}(\mathrm{V})$ building blocks accessible via asymmetric catalysis from commercially available materials. Using the synthetic strategies outlined herein, these versatile intermediates are expected to enable the facile synthesis of both known and new stereogenic-at-P(V) compounds of interest.

\section{REFERENCES}

1. U. Pradere, E. C. Garnier-Amblard, S. J. Coats, F. Amblard, R. F. Schinazi, Synthesis of Nucleoside Phosphate and Phosphonate Prodrugs. Chem. Rev. 114, 9154-9218 (2014).

2. M. D. Sørensen, L. K. A. Blæhr, M. K. Christensen, T. Høyer, S. Latini, P.-J. V. Hjarnaa, F. Björkling, Cyclic phosphinamides and phosphonamides, novel series of potent matrix metalloproteinase inhibitors with antitumour activity. Bioorg. Med. Chem. 11, 5461-5484 (2003).

3. M. Sawa, T. Kiyoi, K. Kurokawa, H. Kumihara, M. Yamamoto, T. Miyasaka, Y. Ito, R. Hirayama, T. Inoue, Y. Kirii, E. Nishiwaki, H. Ohmoto, Y. Maeda, E. Ishibushi, Y. Inoue, K. Yoshino, H. Kondo, New Type of Metalloproteinase Inhibitor: Design and Synthesis of New Phosphonamide-Based Hydroxamic Acids. J. Med. Chem. 45, 919-929 (2002).

4. A. Babbs, A. Berg, M. Chatzopoulou, K. E. Davies, S. G. Davies, B. Edwards, D. J. Elsey, E. Emer, A. L. A. Figuccia, A. M. Fletcher, S. Guiraud, S. Harriman, L. Moir, N. Robinson, J. A. Rowley, A. J. Russell, S. E. Squire, J. E. Thomson, J. M. Tinsley, F. X. Wilson, G. M. 
Wynne, Synthesis of SMT022357 enantiomers and in vivo evaluation in a Duchenne muscular dystrophy mouse model. Tetrahedron. 76, 130819 (2020).

5. A. Nocentini, V. Alterio, S. Bua, L. Micheli, D. Esposito, M. Buonanno, G. Bartolucci, S. M. Osman, Z. A. ALOthman, R. Cirilli, M. Pierini, S. M. Monti, L. Di Cesare Mannelli, P. Gratteri, C. Ghelardini, G. De Simone, C. T. Supuran, Phenyl(thio)phosphon(amid)ate Benzenesulfonamides as Potent and Selective Inhibitors of Human Carbonic Anhydrases II and VII Counteract Allodynia in a Mouse Model of Oxaliplatin-Induced Neuropathy. $J$. Med. Chem. 63, 5185-5200 (2020).

6. A. Nocentini, P. Gratteri, C. T. Supuran, Phosphorus versus Sulfur: Discovery of Benzenephosphonamidates as Versatile Sulfonamide-Mimic Chemotypes Acting as Carbonic Anhydrase Inhibitors. Chem. - Eur. J. 25, 1188-1192 (2019).

7. W. A. Lee, G.-X. He, E. Eisenberg, T. Cihlar, S. Swaminathan, A. Mulato, K. C. Cundy, Selective intracellular activation of a novel prodrug of the human immunodeficiency virus reverse transcriptase inhibitor tenofovir leads to preferential distribution and accumulation in lymphatic tissue. Antimicrob. Agents Chemother. 49, 1898-1906 (2005).

8. T. Imamoto, Synthesis and applications of high-performance P-chiral phosphine ligands. Proc. Jpn. Acad. Ser. B Phys. Biol. Sci. 97, 520-542 (2021).

9. M. Dutartre, J. Bayardon, S. Jugé, Applications and stereoselective syntheses of Pchirogenic phosphorus compounds. Chem. Soc. Rev. 45, 5771-5794 (2016).

10. O. I. Kolodiazhnyi, Phosphorus Compounds of Natural Origin: Prebiotic, Stereochemistry, Application. Symmetry. 13, 889 (2021).

11. O. I. Kolodiazhnyi, A. Kolodiazhna, Nucleophilic substitution at phosphorus: stereochemistry and mechanisms. Tetrahedron Asymmetry. 28, 1651-1674 (2017).

12. O. I. Kolodiazhnyi, Recent developments in the asymmetric synthesis of P-chiral phosphorus compounds. Tetrahedron Asymmetry. 23, 1-46 (2012).

13. X. Ye, L. Peng, X. Bao, C.-H. Tan, H. Wang, Recent developments in highly efficient construction of P-stereogenic centers. Green Synth. Catal. 2, 6-18 (2021).

14. S. Jugé, J. P. Genet, Asymmetric synthesis of phosphinates, phosphine oxides and phosphines by Michaelis Arbuzov rearrangement of chiral oxazaphospholidine. Tetrahedron Lett. 30, 2783-2786 (1989).

15. S. Jugé, M. Stephan, J. A. Laffitte, J. P. Genet, Efficient asymmetric synthesis of optically pure tertiary mono and diphosphine ligands. Tetrahedron Lett. 31, 6357-6360 (1990).

16. T. Koizumi, R. Yanada(nee Ishizaka), H. Takagi, H. Hirai, E. Yoshii, Grignard reaction of 2-phenyl-tetrahydropyrrolo-1,5,2-oxazaphospholes, observation of the stereospecific inversion of configuration. Tetrahedron Lett. 22, 571-572 (1981). 
17. Z. S. Han, N. Goyal, M. A. Herbage, J. D. Sieber, B. Qu, Y. Xu, Z. Li, J. T. Reeves, J.-N. Desrosiers, S. Ma, N. Grinberg, H. Lee, H. P. R. Mangunuru, Y. Zhang, D. Krishnamurthy, B. Z. Lu, J. J. Song, G. Wang, C. H. Senanayake, Efficient Asymmetric Synthesis of PChiral Phosphine Oxides via Properly Designed and Activated Benzoxazaphosphinine-2oxide Agents. J. Am. Chem. Soc. 135, 2474-2477 (2013).

18. K. Kuwabara, Y. Maekawa, M. Minoura, T. Maruyama, T. Murai, Chemoselective and Stereoselective Alcoholysis of Binaphthyl Phosphonothioates: Straightforward Access to Both Stereoisomers of Biologically Relevant $P$-Stereogenic Phosphonothioates. J. Org. Chem. 85, 14446-14455 (2020).

19. A. Mondal, N. O. Thiel, R. Dorel, B. L. Feringa, P-chirogenic phosphorus compounds by stereoselective Pd-catalysed arylation of phosphoramidites. Nat. Catal., 1-10 (2021).

20. E. J. Corey, Z. Chen, G. J. Tanoury, A new and highly enantioselective synthetic route to Pchiral phosphines and diphosphines. J. Am. Chem. Soc. 115, 11000-11001 (1993).

21. K. W. Knouse, J. N. deGruyter, M. A. Schmidt, B. Zheng, J. C. Vantourout, C. Kingston, S. E. Mercer, I. M. Mcdonald, R. E. Olson, Y. Zhu, C. Hang, J. Zhu, C. Yuan, Q. Wang, P. Park, M. D. Eastgate, P. S. Baran, Unlocking P(V): Reagents for chiral phosphorothioate synthesis. Science. 361, 1234-1238 (2018).

22. D. Xu, N. Rivas-Bascón, N. M. Padial, K. W. Knouse, B. Zheng, J. C. Vantourout, M. A. Schmidt, M. D. Eastgate, P. S. Baran, Enantiodivergent Formation of C-P Bonds: Synthesis of P-Chiral Phosphines and Methylphosphonate Oligonucleotides. J. Am. Chem. Soc. 142, 5785-5792 (2020).

23. D. A. DiRocco, Y. Ji, E. C. Sherer, A. Klapars, M. Reibarkh, J. Dropinski, R. Mathew, P. Maligres, A. M. Hyde, J. Limanto, A. Brunskill, R. T. Ruck, L.-C. Campeau, I. W. Davies, A multifunctional catalyst that stereoselectively assembles prodrugs. Science. 356, 426-430 (2017).

24. A. L. Featherston, Y. Kwon, M. M. Pompeo, O. D. Engl, D. K. Leahy, S. J. Miller, Catalytic asymmetric and stereodivergent oligonucleotide synthesis. Science. 371, 702-707 (2021).

25. M. Formica, T. Rogova, H. Shi, N. Sahara, A. J. M. Farley, K. E. Christensen, F. Duarte, D. J. Dixon, Catalytic Enantioselective Nucleophilic Desymmetrisation of Phosphonate Esters (2021), doi:10.33774/chemrxiv-2021-5714s.

26. C. Bauduin, D. Moulin, E. B. Kaloun, C. Darcel, S. Jugé, Highly Enantiomerically Enriched Chlorophosphine Boranes: Synthesis and Applications as P-Chirogenic Electrophilic Blocks. J. Org. Chem. 68, 4293-4301 (2003).

27. T. Kimura, T. Murai, Enantiomerically pure P-chiral phosphinoselenoic chlorides: inversion of configuration at the P-chirogenic center in the synthesis and reaction of these substances. Chem. Commun., 4077-4079 (2005). 
28. T. Kimura, T. Murai, P-Chiral Phosphinoselenoic Chlorides and Optically Active P-Chiral Phosphinoselenoic Amides: Synthesis and Stereospecific Interconversion with Extrusion and Addition Reactions of the Selenium Atom. Chem. Lett. 33, 878-879 (2004).

29. A. G. Doyle, E. N. Jacobsen, Small-Molecule H-Bond Donors in Asymmetric Catalysis. Chem. Rev. 107, 5713-5743 (2007).

30. D. A. Kutateladze, D. A. Strassfeld, E. N. Jacobsen, Enantioselective Tail-to-Head Cyclizations Catalyzed by Dual-Hydrogen-Bond Donors. J. Am. Chem. Soc. 142, 69516956 (2020).

31. A. J. Bendelsmith, S. C. Kim, M. Wasa, S. P. Roche, E. N. Jacobsen, Enantioselective Synthesis of $\alpha$-Allyl Amino Esters via Hydrogen-Bond-Donor Catalysis. J. Am. Chem. Soc. 141, 11414-11419 (2019).

32. D. D. Ford, D. Lehnherr, C. R. Kennedy, E. N. Jacobsen, Anion-Abstraction Catalysis: The Cooperative Mechanism of $\alpha$-Chloroether Activation by Dual Hydrogen-Bond Donors. ACS Catal. 6, 4616-4620 (2016).

33. K. L. Tan, E. N. Jacobsen, Indium-Mediated Asymmetric Allylation of Acylhydrazones Using a Chiral Urea Catalyst. Angew. Chem. Int. Ed. 46, 1315-1317 (2007).

34. H. Xu, S. J. Zuend, M. G. Woll, Y. Tao, E. N. Jacobsen, Asymmetric Cooperative Catalysis of Strong Brønsted Acid-Promoted Reactions Using Chiral Ureas. Science. 327, 986-990 (2010).

35. D. A. Strassfeld, E. N. Jacobsen "The Aryl-Pyrrolidine-tert-Leucine Motif as a New Privileged Chiral Scaffold: The Role of Noncovalent Stabilizing Interactions" in Supramolecular Catalysis: New Directions and Developments, P.W.N.M. van Leeuwen, M. Raynal, Eds. (Wiley, 2022), chap. 25, pp. 361-385.

36. I. V. L. Wilkinson, K. J. Perkins, H. Dugdale, L. Moir, A. Vuorinen, M. Chatzopoulou, S. E. Squire, S. Monecke, A. Lomow, M. Geese, P. D. Charles, P. Burch, J. M. Tinsley, G. M. Wynne, S. G. Davies, F. X. Wilson, F. Rastinejad, S. Mohammed, K. E. Davies, A. J. Russell, Chemical Proteomics and Phenotypic Profiling Identifies the Aryl Hydrocarbon Receptor as a Molecular Target of the Utrophin Modulator Ezutromid. Angew. Chem. Int. Ed. 59, 2420-2428 (2020).

37. A. Babbs, A. Berg, M. Chatzopoulou, K. E. Davies, S. G. Davies, B. Edwards, D. J. Elsey, E. Emer, S. Guiraud, S. Harriman, C. Lecci, L. Moir, D. Peters, N. Robinson, J. A. Rowley, A. J. Russell, S. E. Squire, J. M. Tinsley, F. X. Wilson, G. M. Wynne, 2Arylbenzo[d]oxazole Phosphinate Esters as Second-Generation Modulators of Utrophin for the Treatment of Duchenne Muscular Dystrophy. J. Med. Chem. 63, 7880-7891 (2020).

38. M. Chatzopoulou, E. Emer, C. Lecci, J. A. Rowley, A.-S. Casagrande, L. Moir, S. E. Squire, S. G. Davies, S. Harriman, G. M. Wynne, F. X. Wilson, K. E. Davies, A. J. Russell, Decreasing HepG2 Cytotoxicity by Lowering the Lipophilicity of 
Benzo[d]oxazolephosphinate Ester Utrophin Modulators. ACS Med. Chem. Lett. 11, 24212427 (2020).

\section{Acknowledgments}

We thank Dr. S.-L. Zheng (Harvard University) for determination of the X-ray crystal structure, and R. Algera, J. Essman, and H. Sharma for helpful discussions. Funding: National Institutes of Health grant GM043214 (ENJ). Crystallographic data for compound 10 is available free of charge from the Cambridge Crystallographic Data Centre under reference CCDC 2155524. All other data are available in the main text or the supplementary materials.

\section{Supplementary Materials}

Materials and Methods

Supplementary Text

Figs. S1 and S2

Tables S1 to S7

References (39-56) 